\title{
Possible Involvement of Liver Resident Macrophages (Kupffer Cells) in the Pathogenesis of Both Intrahepatic and Extrahepatic Inflammation
}

\author{
Yuki Kakinuma, Takuya Kimura, and Yoshifumi Watanabe \\ Department of Pharmaceutical Sciences, Musashino University, Tokyo 202-0023, Japan \\ Correspondence should be addressed to Yoshifumi Watanabe; ywatanab@musashino-u.ac.jp
}

Received 2 June 2017; Accepted 27 June 2017; Published 19 July 2017

Academic Editor: Pierluigi Toniutto

Copyright (C) 2017 Yuki Kakinuma et al. This is an open access article distributed under the Creative Commons Attribution License, which permits unrestricted use, distribution, and reproduction in any medium, provided the original work is properly cited.

Liver resident macrophages designated Kupffer cells (KCs) form the largest subpopulation of tissue macrophages. KCs are involved in the pathogenesis of liver inflammation. However, the role of KCs in the systemic inflammation is still elusive. In this study, we examined whether KCs are involved in not only intrahepatic inflammation but also extrahepatic systemic inflammation. Administration of clodronate liposomes resulted in the KC deletion and in the suppression of liver injury in T cell-mediated hepatitis by ConA as a local acute inflammation model, while the treatment did not influence dextran sulfate sodium- (DSS-) induced colitis featured by weight loss, intestinal shrink, and pathological observation as an ectopic local acute inflammation model. In contrast, KC deletion inhibited collagen-induced arthritis as a model of extrahepatic, systemic chronical inflammation. KC deleted mice showed weaker arthritic scores, less joint swelling, and more joint space compared to arthritis-induced control mice. These results strongly suggest that KCs are involved in not only intrahepatic inflammatory response but also systemic (especially) chronic inflammation.

\section{Introduction}

Kupffer cells (KCs) are liver resident macrophages which represent the largest population of macrophages in the body, constituting approximately $10 \%$ of all hepatic cells and approximately $80 \%$ of the tissue macrophages, including alveolar, splenic, and peritoneal macrophages [1]. KC has been a mysterious population for long time because the features including specific markers and the functions have been elusive, and only the designation "liver resident macrophage" has been established. Recently, some researchers tried to find and report the specific markers of the population [2-7]. These markers are becoming powerful tools to identify this population. Compared to specific markers, the functions have been extensively investigated, especially (and naturally) in the field of liver inflammation [8-14]. For these studies, one important finding by Van Rooijen and Sanders was the key triggering method that intravenous administration of clodronatecontaining liposomes specifically deletes spleen and liver but not other tissue resident macrophages [15]. However, the attention of the researchers have been restricted only to the functions of Kupffer cells in the specific local site, liver. We speculated that this largest population of tissue macrophages has influence on systemic inflammation in some way. In this study, we examined the role of Kupffer cells in local, systemic, acute, and chronic inflammation models and indicate that Kupffer cells are involved in the pathogenesis of collagen-induced arthritis as a model of chronic, systemic inflammation.

\section{Material and Methods}

2.1. Animals. Female ICR, C57BL6 and DBA/2 mice (6 weeks old) were purchased from Tokyo Laboratory Animals Sciences Co., Ltd. (Tokyo, Japan). All mice were housed in a SPF facility. Water and food were available ad libitum. Each in vivo experiment for pathological models was conducted with 7-10 mice per group. All animal experiments were conducted in accordance with local institutional guidelines for the care and use of laboratory animals. 


\subsection{Animal Models}

2.2.1. ConA Hepatitis. ConA hepatitis was induced as described [16]. Briefly, ConA (10 mg/kg) in PBS was intravenously injected into the tail vein of ICR mic;, GPT and GOT in the plasma were measured 3,12 , and $24 \mathrm{~h}$ after treatment.

2.2.2. Dextran Sulfate- (DSS-) Induced Colitis. Colitis was induced by the dietary administration of $4 \%$ DSS solution for 7 days via drinking water ad libitum and then back to water without DSS in C57BL/6 mice as described [17]. DSS was obtained from Wako Chemicals, Ltd. (Tokyo, Japan). Mice were inspected daily and the body weights were measured daily and they were scarified 10 days after the start of pathological inspection.

2.2.3. Collagen-Induced Arthritis. CIA was induced by the administration of $100 \mathrm{ug}$ collagen type II emulsified with complete Freund's adjuvant ( $70 \mathrm{ul}$ ) as the first immunization, followed by the second immunization with the same amount of collagen in incomplete Freund's adjuvant as described in detail [18]. The number of arthritic limbs were quantitated and each limb was assigned a severity score of $0-4$ according to the criteria in [18].

2.3. Kupffer Cell Deletion. Kupffer cells were deleted from the liver by the intravenous administration of clodronate liposomes as described [19]. Clodronate liposomes were obtained from Katayama Chemicals, Ltd. (Osaka, Japan).

2.4. Cell Preparation. KCs were prepared by collagenase liver perfusion and differential density centrifugation. Briefly, the liver was perfused in situ with a HEPES based buffer containing $0.015 \%$ collagenase (Sigma) and $0.004 \%$ trypsin inhibitor (Wako Pure Chemical Industries Ltd., Osaka, Japan). After excision, the hepatic cells were suspended in Hanks' balanced salt solution and filtered through a nylon mesh. The filtrate was centrifuged thrice at $50 \times \mathrm{g}\left(4^{\circ} \mathrm{C}\right)$ for $90 \mathrm{~s}$ to delete the parenchymal hepatocytes. Flow cytometric analysis involved centrifugation of purified fractions of nonparenchymal cells (NPC) from the liver, on a layer of $20 \%$ and $50 \%$ Percoll, at $1,000 \times \mathrm{g}\left(4^{\circ} \mathrm{C}\right)$ for $20 \mathrm{~min}$ in order to remove dead cells. The suspension containing KCs was collected from the intermediate layer and resuspended in PBS containing $5 \mathrm{mM}$ EDTA and 1\% FBS, for antibody binding as described [7].

2.5. cDNA Synthesis, DNA Analysis, and RT-PCR. Total RNA was extracted from the cells using TRIzol Reagent (Invitrogen, Carlsbad, CA, USA), according to the manufacturer's instructions. Complementary DNA was synthesized using the SuperScript ${ }^{\mathrm{TM}}$ III Cells Direct cDNA Synthesis System (Invitrogen). Complementary RNA amplification, labeling, hybridization, and analysis, between the KCs and PEC macrophages, were performed by Toray Research Institute, Co. Ltd. (Kamakura, Japan). Saturation of PCR products was avoided using 20 PCR cycles during amplification. The PCR reactions were carried out using a three-step protocol: $94^{\circ} \mathrm{C}$ for $15 \mathrm{~s}$, annealing at $55^{\circ} \mathrm{C}$ for $15 \mathrm{~s}$, followed by $72^{\circ} \mathrm{C}$ for $30 \mathrm{~s}$. Actin or GAPDH was used as an internal reference. The PCR products were analyzed by $2 \%$ agarose gel electrophoresis. The primers employed in the experiments are described [20].

2.6. Flow Cytometry Analysis. Nonspecific binding of immunoglobulin to the Fc receptors was blocked by preincubating $200 \mu \mathrm{l}$ of cell suspension, adjusted to $5 \times 10^{6} \mathrm{cells} / \mathrm{ml}$, with $25 \mathrm{ng} / \mathrm{ml}$ unlabeled murine polyclonal IgG antibody and $2.5 \mathrm{ng} / \mathrm{ml}$ anti-mouse CD16/32 antibody (BioLegend), at $4^{\circ} \mathrm{C}$ for $10 \mathrm{~min}$. Cell surface molecules were stained with the primary antibody, followed by the labeled-secondary antibody or $2.5 \mathrm{ng} / \mathrm{ml}$ fluorescent-labeled antibodies, at $4^{\circ} \mathrm{C}$ for $20 \mathrm{~min}$. After washing with ice-cold PBS, cells were resuspended in up to $300 \mu \mathrm{l}$ of sheath fluid containing $5 \mathrm{ng} / \mathrm{ml}$ propidium iodide, in order to identify the dead cells. Data acquisition and analysis were performed on the BD FACSAriaTM II flow cytometer (Becton, Dickinson and Company, Franklin Lakes, NJ, USA). The results in manuscript were the representatives of three or four experiments.

2.7. MTT Assay. The cell number of treated cells was evaluated by MTT assay [21]. Briefly, MTT was added to treated cells plated in a 96-well plate at a final concentration of $500 \mathrm{ug} / \mathrm{ml}$, and the cells were incubated for $4 \mathrm{~h}$ at $37^{\circ} \mathrm{C}$. Then $100 \mathrm{ul}$ of acidic isopropyl alcohol was added to each well. The solution was vigorously mixed to solubilize the reacted dye. The absorbance of each well at $550 \mathrm{~nm}$ was measured using the microplate reader.

2.8. Histological Analysis. Tissues were dissected and fixed overnight in $4 \%$ paraformaldehyde solution at $4^{\circ} \mathrm{C}$. Tissues were embedded in Tissue-Tek compound (Miles Laboratories, Elkhart, IN) and frozen in liquid nitrogen for preparation of cryostat sections. All sections $(10 \mu \mathrm{m})$ were lightly counterstained with $0.1 \%$ hematoxylin and eosin [22].

2.9. Statistical Analysis. The data are representative of three or four experiments. Experiments were performed in triplicate, and the error bars are the mean standard deviation (SD) of a single experiment. Differences between groups were examined for statistical significance using one-way ANOVA followed by the Student-Newman-Keul test. A $P$ value of less than 0.05 denoted the presence of a statistically significant difference.

\section{Results}

3.1. Treatment with Clodronate Liposome Deletes Kupffer Cells but Does Not Affect Other Cell Populations. As already known, administration of clodronate-encapsulated liposomes deletes Kupffer cells (KCs) in vivo [15]. However, the effective duration time and influence on other cell types are not well understood. We first examined the duration time of $\mathrm{KC}$ deletion in the liver and the changes of other cell populations after treatment (Figure 1). The population of KCs was expressed as $\mathrm{F} 4 / 80^{+} \mathrm{CD} 11 \mathrm{~b}^{\text {middle+ }}$ group (Figure $1(\mathrm{a})$ ) and the population was completely deleted by clodronate-liposome administration for 5 days after treatment and then gradually restored from 7 to 9 days (Figure 1(a)). In contrast, the treatment did not show any significant influence 

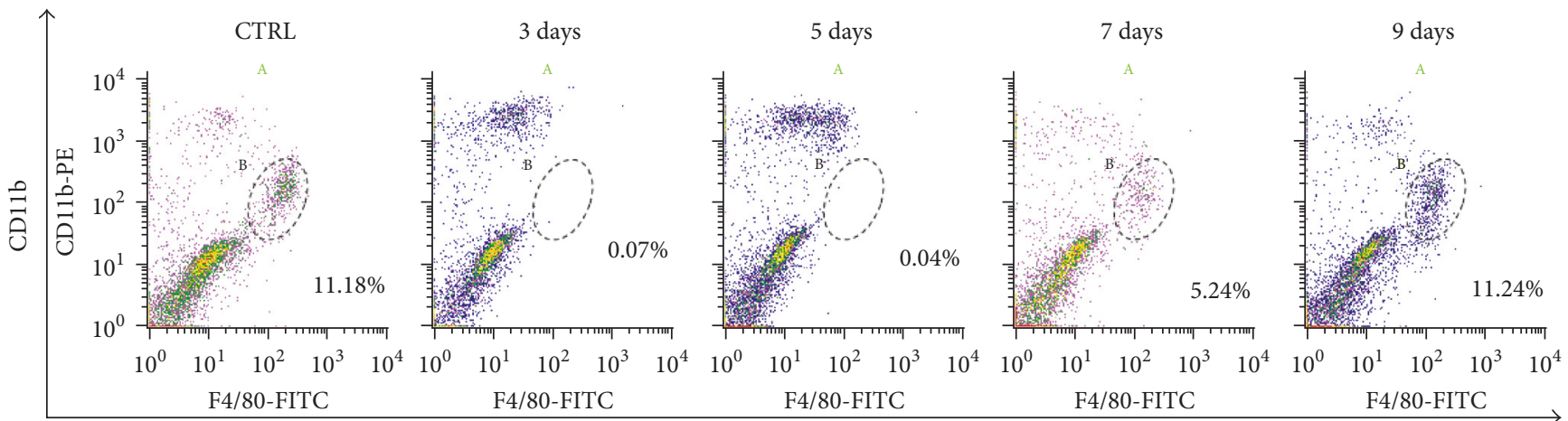

F4/80

(a)

CTRL

LEC
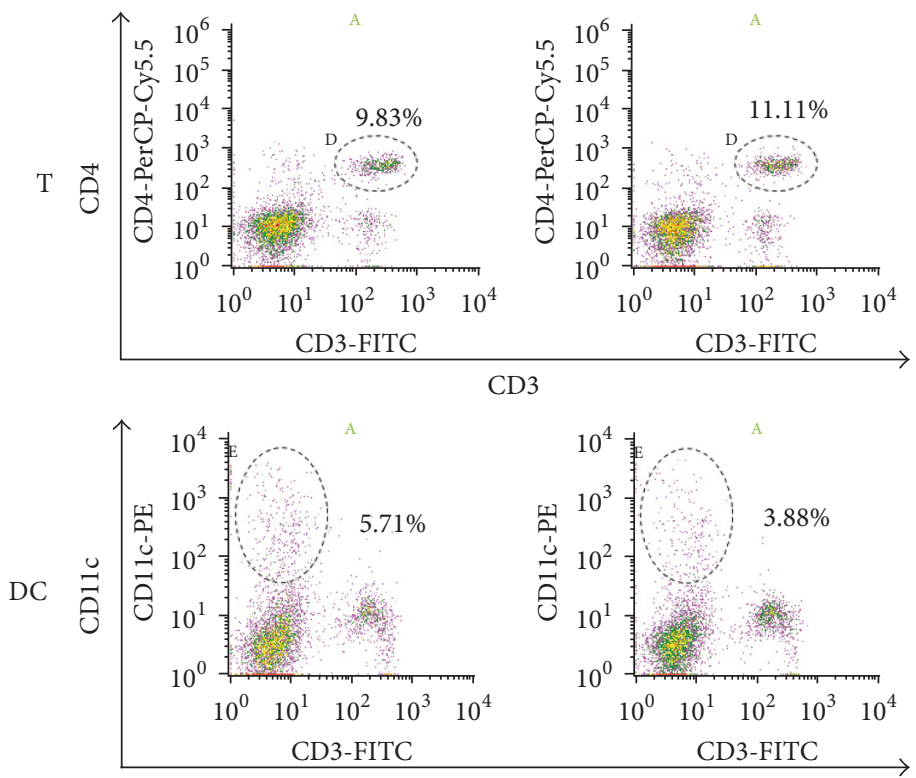

CD3

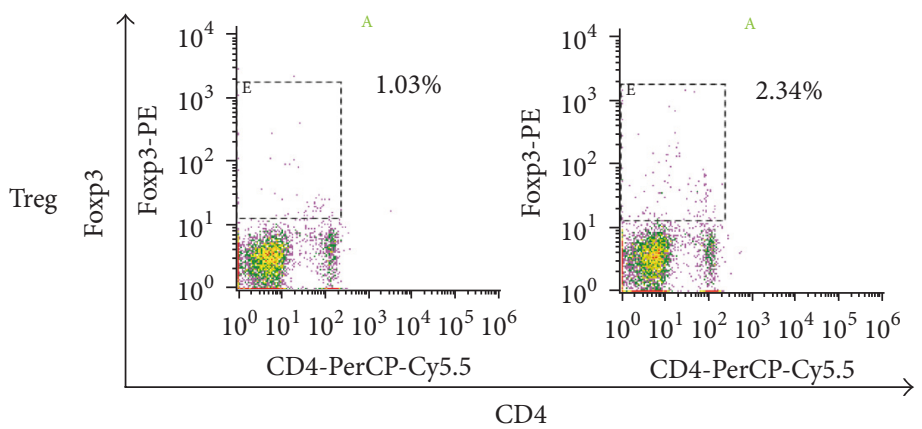

(b)

FIGURE 1: Treatment with clodronate liposome deletes Kupffer cells but does not affect other cell populations. DBA/2 mice were intravenously administered with $100 \mathrm{ul}$ of clodronate liposome. (a) Nonparenchymal cells from the livers were collected by collagenase infusion described in Material and Methods at indicated days after treatment and then subjected to flow cytometry analysis using anti-F4/80 and anti-CD11b antibodies. The population of Kupffer cells was indicated as F4/80 positive and CD1lb middle positive population. (b) Splenocytes from control or treated mice 3 days after treatment were collected and subjected to flow cytometry analysis for helper T cells $\left(\mathrm{CD}^{+} \mathrm{CD}^{+}\right)$, dendritic cells (DC) $\left(\mathrm{CD}^{-} \mathrm{CD}^{-11 b^{+}}\right)$, and regulatory T cells $\left(\mathrm{CD}^{+} \mathrm{Foxp}^{+}\right)$. A, B, D, and $\mathrm{E}$ are target populations. 


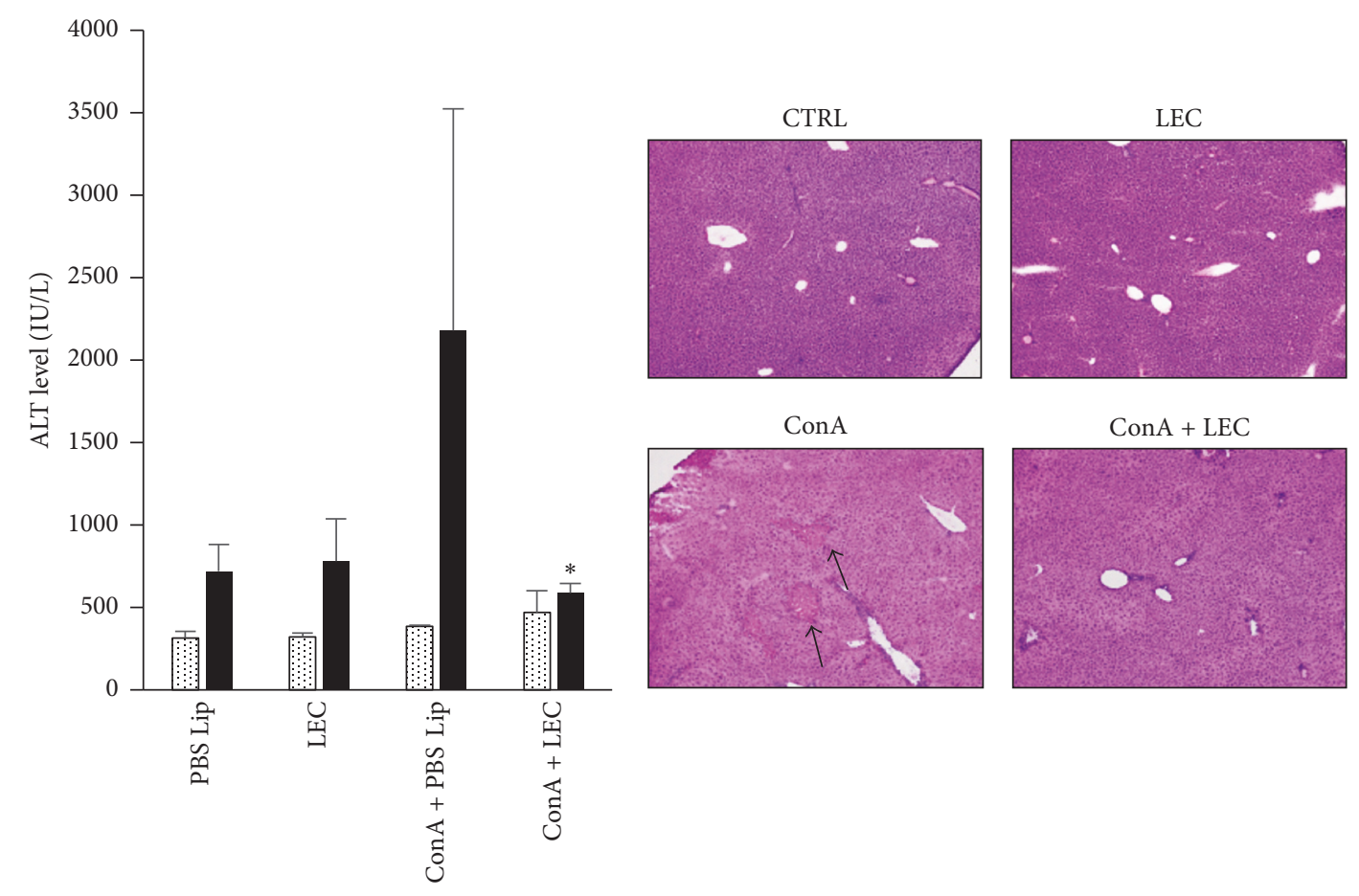

(a)

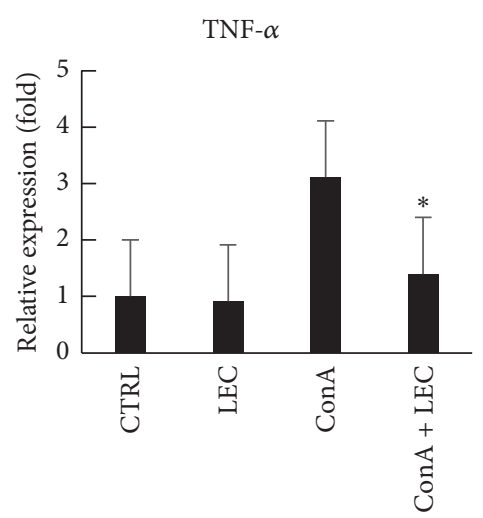

(b)

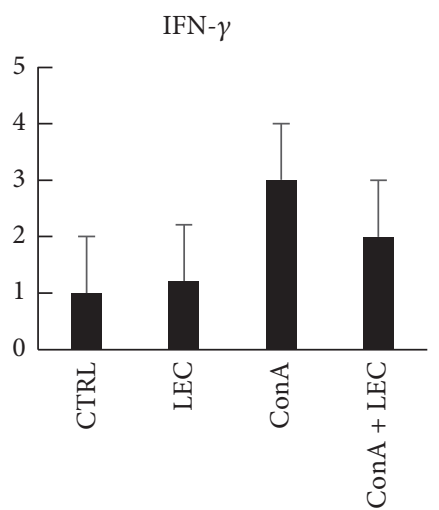

(c)

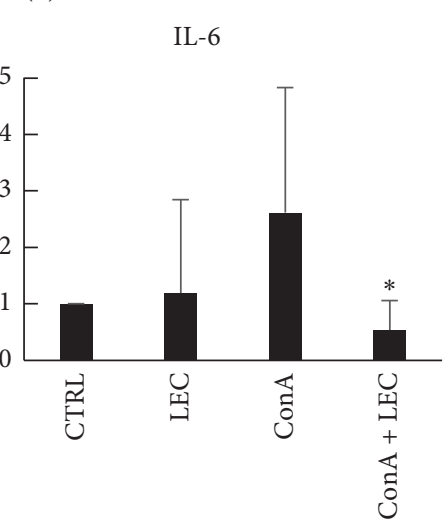

FIGURE 2: Deletion of Kupffer cells suppresses ConA-induced hepatitis. Con A ( $27.5 \mathrm{mg} / \mathrm{kg})$ was intravenously injected into control or clodronateliposome (Clp) treated mice 3 days after liposome treatment. Mice were dissected $24 \mathrm{~h}$ after ConA treatment and the ATL activities in the serum were measured (a) and the livers were subjected to histological analysis (b). Real-time PCR was performed using cDNA from the liver in the treated mice for the quantification of IFN $-\gamma$, TNF- $\alpha$, and IL-6 mRNA. ${ }^{*} P<0.01$.

on the population of helper $\mathrm{T}$ cells $\left(\mathrm{CD} 3^{+} \mathrm{CD} 4^{+}\right)$, DCs $\left(\mathrm{CD}^{-} \mathrm{CD} 1 \mathrm{c}^{+}\right)$, or regulatory $\mathrm{T}$ cells $\left(\right.$ Foxp $\left.^{+}\right)$(Figure $\left.1(\mathrm{~b})\right)$. These results show that clodronate liposomes induce selective but temporal deletion of KCs.

3.2. Deletion of Kupffer Cells Suppresses Local Acute Inflammation in the Liver. To examine the role of KCs in local acute inflammation, we investigated T cell-mediated acute hepatitis model induced by concanavalin A (ConA). ConA induced liver injury within $24 \mathrm{~h}$ after administration (Figure 2(a)) and the deletion of KCs completely suppressed the hepatitis in the aspects of ALT and tissue section observation (Figures 2(a) and 2(b)). Deletion of KCs resulted in the inhibition of inflammatory cytokine mRNA expression such as IFN$\gamma, \mathrm{TNF} \alpha$, and IL-6. In particular, suppression of TNF $\alpha$ and IL-6 mRNA expression were greater than IFN- $\gamma$. Thus, it is assumed that TNF $\alpha$ and IL- 6 produced by KCs play a significant role in the pathogenesis of the hepatitis.

3.3. Kupffer Cells Are Not Involved in the Pathogenesis of Chemically Induced Extrahepatic Acute Inflammation. Next, we examined the influence of $\mathrm{KC}$ deletion on dextran sodium 


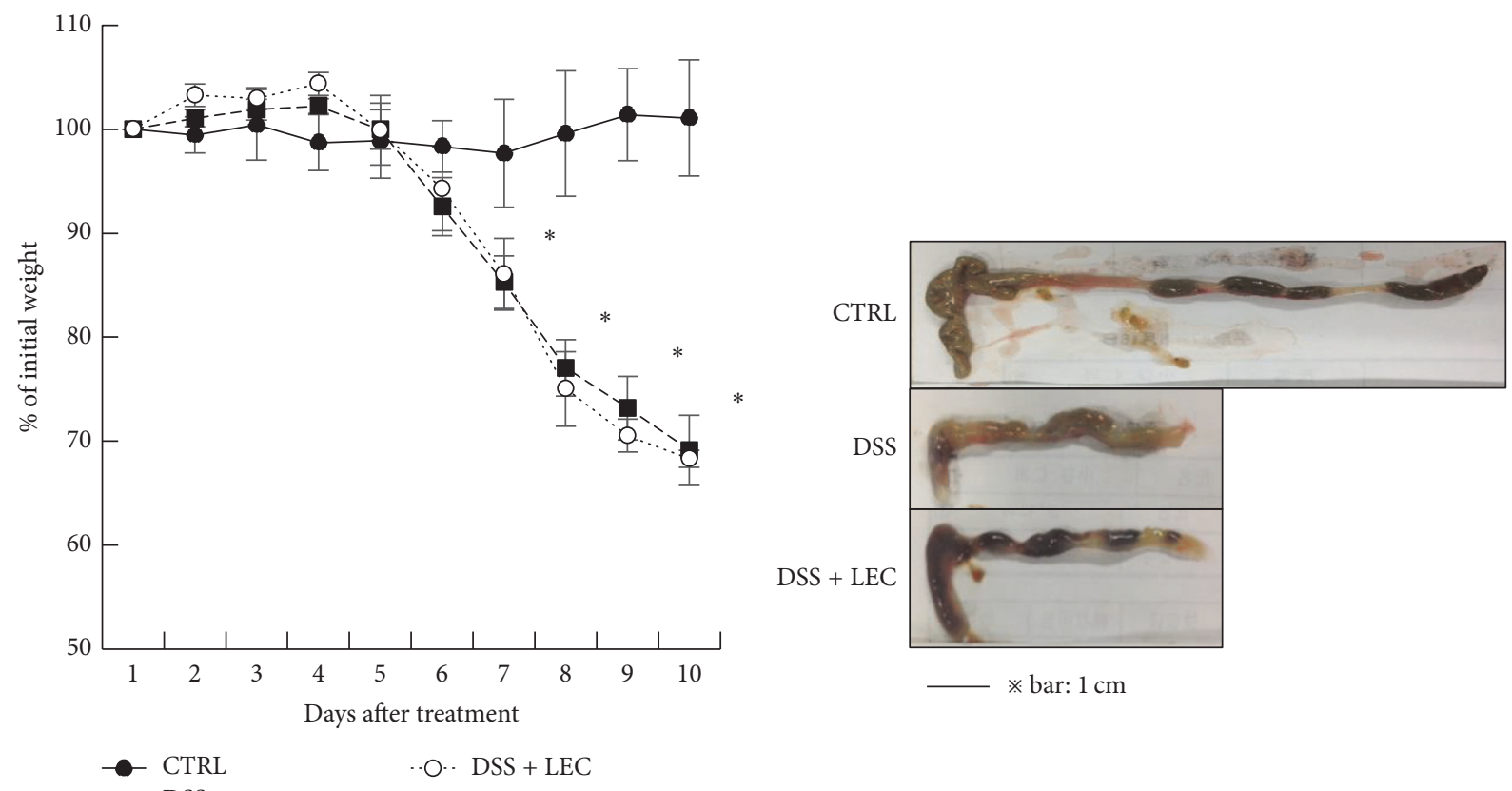

(a)

CTRL

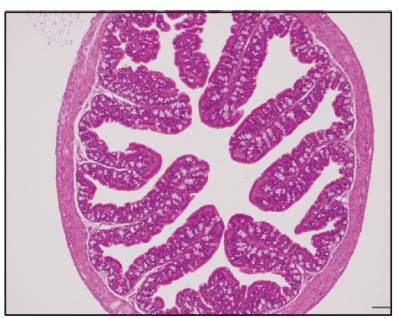

DSS

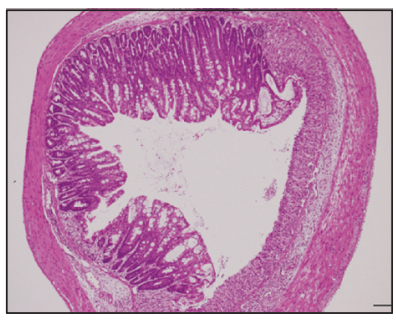

(c) (b)

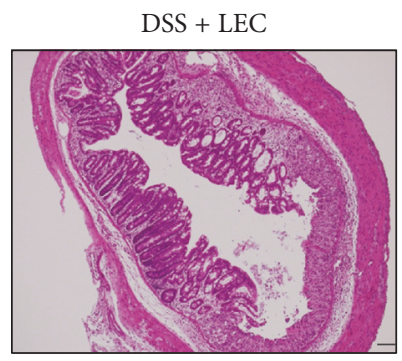

FIGURE 3: DSS-induced colitis was not suppressed by the deletion of Kupffer cells. C57BL/6 mice were treated with 100 ul of clodronate liposome (LEC) $24 \mathrm{~h}$ before DSS administration. The mice were administered 3\% DSS in the drinking water ad libitum for 7 days and then back to water without DSS for 3 days. Mice were subjected to pathological analysis. (a) Daily weight changes of treated mice. (b) Comparison of intestines from treated mice. (c) Histological analysis of the samples in (b). CTRL: control; DSS: DSS treated; DSS + LEC: DSS treated in KC deleted mice. ${ }^{*} P<0.01$.

sulfate- (DSS-) induced colitis as a model of extrahepatic acute inflammation, which shows features of ulcerative colitis with inflammation and exhibits features of Crohn's disease such as Thl dependency $[23,24]$. In this model system, KC deletion did not show any significant changes in the features (weight (Figure 3(a)), colon length (Figure 3(b)), and tissue histology (Figure 3(c))). Although DSS induced weight loss because of diarrhea with the shrink of colon, KC deletion did not inhibit the colon injury (Figures 3(a) and 3(b)). DSS also induced colon inflammation with multifocal submucosal oedema and pronounced loss of goblet cells (Figure 3(c)), which was not influenced by the administration of clodronate liposomes.

3.4. Kupffer Cells Are Involved in the Systemic Chronic Inflammation. Finally, we examined the role of KCs in collageninduced arthritis (CIA) as a systemic chronic inflammation model. Different from chemically induced acute colitis, collagen-induced chronic arthritis is mediated by acquired immune reaction [18], which require various kinds of cell types for establishment. The score of arthritic feature increased after the collagen second challenge (Figure 4(b)). In contrast, KC deletion significantly suppressed the score (Figure $4(\mathrm{~b})$ ). In terms of paw swelling, the same tendency was observed in both front and hind paws (Figure 4(c)). Histological features also confirmed the above observation. As shown in Figure 4(d), collagen challenge induced joint swelling and the joint space was scarcely observed compared to control. In contrast, KC deletion suppressed the joint swelling and maintained the joint space (Figure 4(d)). In order to examine the T cell response as the establishment of acquired immunity, the splenocytes from collagen-challenged mice were collected 15 days after the challenge and then stimulated with collagen in vitro. Three days after the in vitro stimulation, the cell number of the samples was measured by MTT assay. Figure 4(e) shows that splenocytes from $\mathrm{KC}$ deleted mice challenged with 
(day)

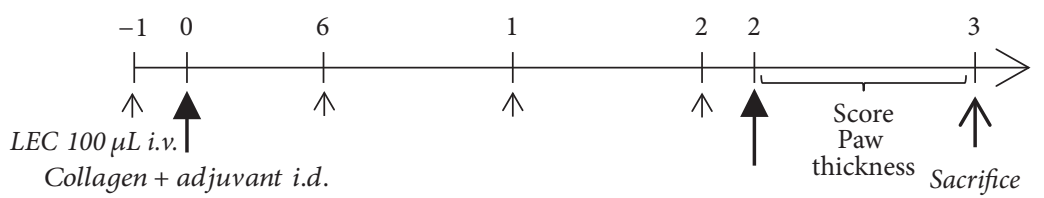

(a)

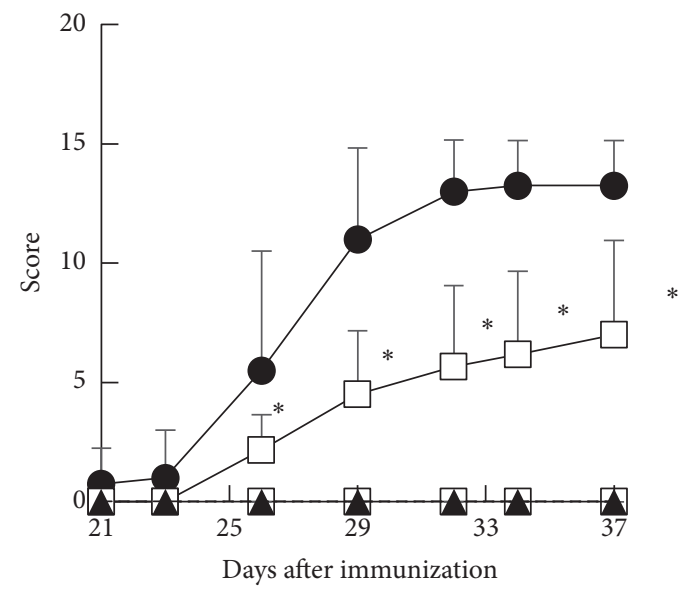

. CTRL

$-\square-$ CIA + LEC

(b)
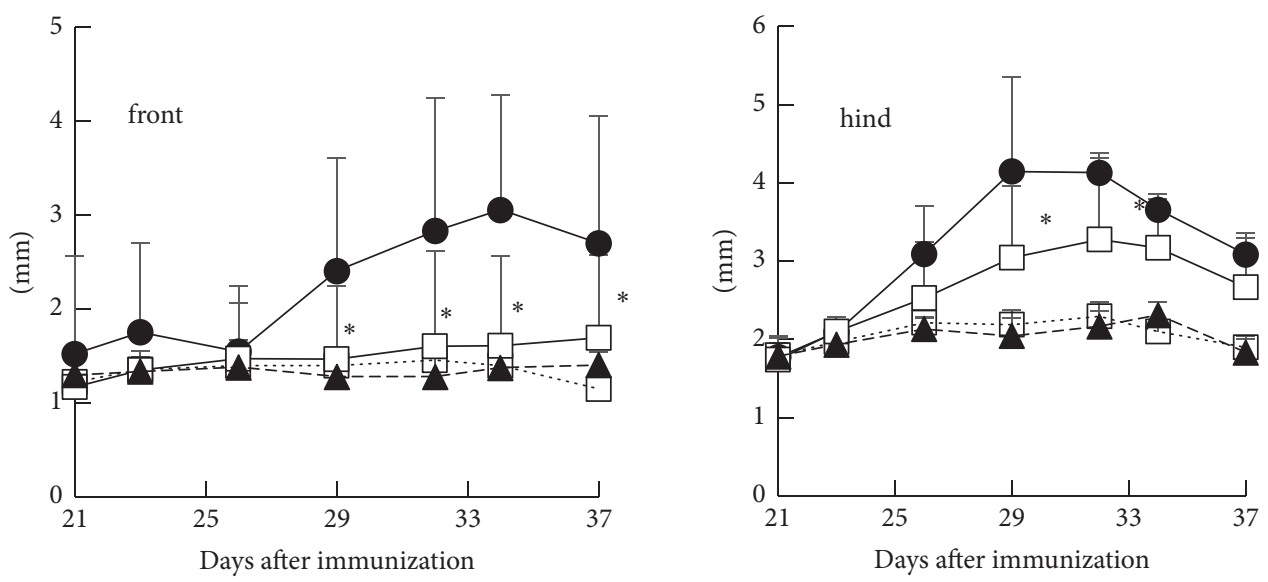

$\begin{array}{ll}\square . \text { CTRL } & - \text { - CIA + LEC } \\ - \text { CIA } & -\mathbf{A}-\text { LEC }\end{array}$
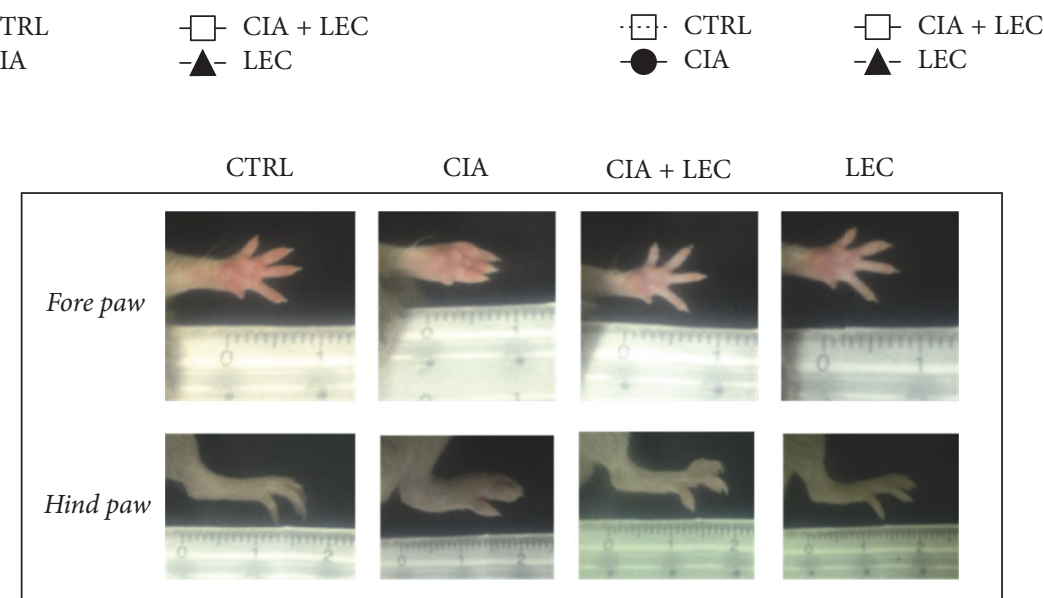

(c)

Figure 4: Continued. 


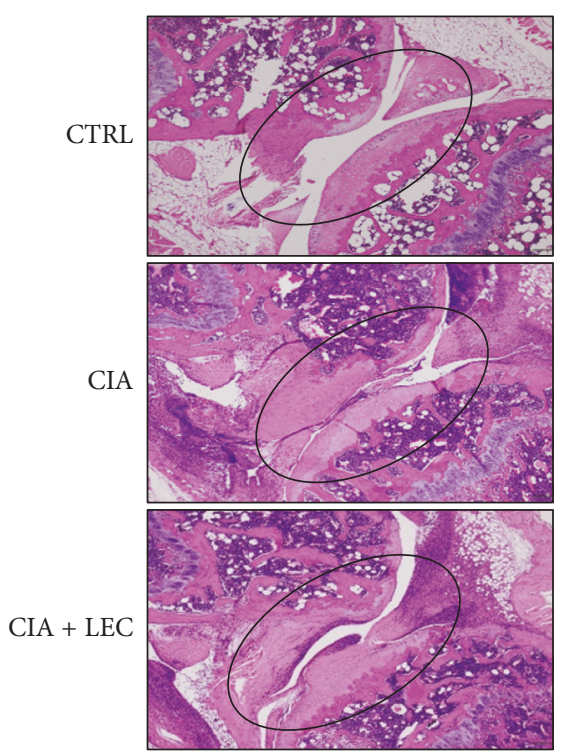

(d)

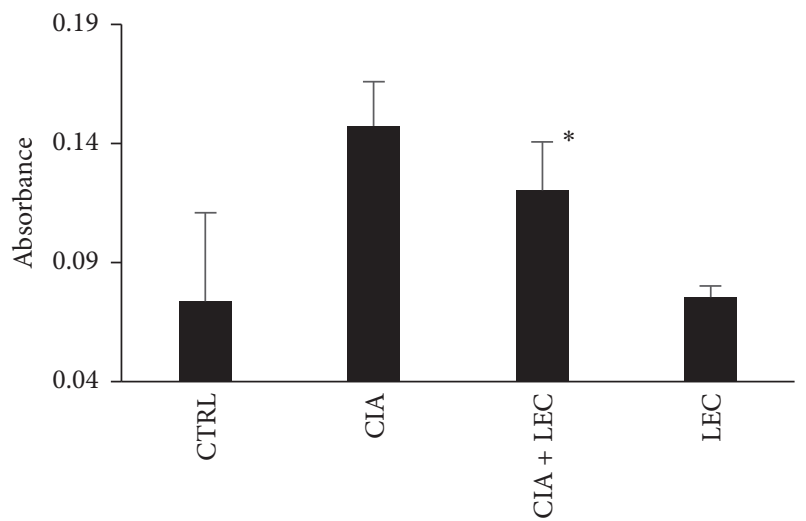

(e)

FIGURE 4: Deletion of Kupffer cells partly regulated collagen-induced arthritis. Collagen-induced arthritis was induced in control or KC deleted DBA/2 mice as scheduled in (a). One hundred ul of clodronate liposome (LEC) was administered every 7 days (small arrows). Collagen was intradermally injected at days 0 and 21 as middle arrows. (b) Severity scores of arthritic limbs from treated mice. The number of arthritic limbs was quantitated and assigned a severity score as described in Material and Methods. ${ }^{*} P<0.01$ (c) Comparison of limbs thickness as the feature of arthritis. ${ }^{*} P<0.01$ (d) Histological analysis of the limb joints from the treated mice. (e) Splenocytes response activity assay. Splenocytes from the sacrificed mice were stimulated in vitro with collagen $(75 \mathrm{ug} / \mathrm{ml})$ for $72 \mathrm{~h}$ and then MTT assay was performed. ${ }^{*} P<0.05$.

collagen exhibited less proliferation ability compared to those from collagen-challenged mice. KC deletion without collagen challenge did not show any significant change as control.

\section{Discussion}

In this study, we show that Kupffer cells (KCs) are involved in the onset of both local (acute hepatitis) and systemic (collagen-induced arthritis) inflammation.

Several studies reported that clodronate liposomes (CLs) suppressed arthritis when prepared with small unilamellar vesicles (SUV) and administered systemically postarthritis induction $[25,26]$ or administered directly into joints [27, 28]. These studies suggest the involvement of synovial tissue macrophages of joints in the progression of arthritis. Both routes of CLs administration deleted or decreased the synovial macrophages in joints and the reduction was correlated with the suppression of arthritis [25-28]. However, systemic administration of CLs did not influence the number of synovial phagocytic cells in normal [15] or inflammatory joints when the liposomes were prepared with multilamellar vesicles (MLV) [25]. Commercially available reagent used in this study is composed of MLVs; thus, the reagent is thought not to influence synovial macrophages in joints. In contrast, it is thought that when the liposomes are prepared with SUV, these nanoparticles could pass through the endothelial fenestrae of blood vessels only in the inflammatory tissues because of enhanced permeability and retention (EPR) effect [29] by cytokines such as VEGF (also designated Vascular Permeability Factor (VPF) because of the function), the major cytokine to induce blood vessel permeability, in inflammation [30]. In fact, it is reported that SUV-CLs are more effective than MLV-CLs to decrease the number of synovial macrophages in inflammatory joints [26]. However, these reports suggest the significance and role of synovial macrophages in joints but not those of KCs which were deleted at the same time. To examine the role of KCs, CLs prepared with MLV to avoid the influence on synovial macrophages should be administered before the onset of arthritis induction.

At present, we do not have any concrete data to explain how KCs are involved in the pathogenesis of arthritis. Basically, KCs are heterogeneous cell populations [31, 32] and show inflammatory phenotypes (M1) in some cases $[9,11,12$, 14,33 ] while they do anti-inflammatory phenotypes (M2) in others [34-38], depending on the circumstance conditions [10]. In this study, KCs played as M1 cells in the onset of arthritis. Phagocytic cells in the synovial membrane of joints are thought to be critical mediators in the inflammation of arthritis according to the previous studies [26-28]. Synovial macrophages comprise two subsets: resident tissue macrophages and infiltrated inflammatory macrophages. It is noteworthy that the phagocyte population involved in the pathogenesis of arthritis expresses complement receptor for $\mathrm{C} 3 \mathrm{~b}$ and iC3b (CRIg) (also designated VSIG4 or Z39Ig) [39, 40], 
which is selectively expressed on tissue macrophages, mainly on $\mathrm{KCs}[3,4]$, and the expression increased according to the severity of the arthritis [40]. The antigen itself functions as anti-inflammatory by inducing tolerance in $\mathrm{T}$ cells rather inflammatory [4]. Thus, it is thought that the increase amount of antigen reflects the increase number of KC-like cells in the synovial membrane of joints. Some studies suggest the similarity of the phagocytic cells in synovial membranes of arthritis joints to KCs in the liver [39, 40]. Alternatively, KCs may increase the number of these synovial phagocytes. Thus, it is assumed that the interaction between KCs and these phagocytic cells in the joints is the key of the mechanism. The possible hypothesis is that cytokine(s) from KCs activates distal phagocytic cells in synovial membranes of joints. Such cytokine(s) is not elucidated, but IL-4 to induce the proliferation of tissue macrophages is a potential candidate [41], or it is suggested that there is a network between KCs and monocytes, in which different macrophage populations communicate, activate, or suppress each other [42]. Recently, Fujiu et al. reported that the existence of homeostasis regulation between distal organs (heart-brainkidney) through the tissue macrophage communication [43]. Although KCs are fully capable of migrating in vitro [7], it is not naturally thought that KCs themselves migrate into joints and induce inflammation. So far, we cannot completely delete the possible involvement of splenic or synovial macrophages in the regulation in spite of the fact that the major population which MLV clodronate liposomes delete is KCs. It is difficult to identify the substantial specific tissue macrophage population because there is not any marker with strictly restricted expression on the specific population of tissue macrophages. This issue is to be solved.

More possible explanation is that KCs promote Th17 differentiation. IL-17 producing T helper cells, Th17 cells, are key cell population in the pathogenesis of various autoimmune and inflammation diseases [44], including autoimmune arthritis $[45,46]$, and these cells play a central role in collagen-induced arthritis [47]. The differentiation of these cells is dependent on the copresence of IL- 6 and TGF- $\beta$ [44]. Interestingly, Kupffer cell is the main cell population to produce TGF- $\beta$ in normal liver $[48,49]$ and these cells also express IL- 6 by the stimulation of inflammation. Thus, it is naturally thought that KCs promote Th17 differentiation. In fact, Xie et al. reported that KCs induce Th17 differentiation at local site (liver). Considering the large size of KC population, these cytokines are thought to regulate the Th17 differentiation in distal sites. Sasai et al. reported that IL-6 promoted the onset of CIA [50]. The hypothesis that KCs promote Th17 differentiation in synovial joints by producing TGF $\beta$ and IL- 6 is under investigation.

Deletion of KCs did not suppress DSS-induced colitis in all features of colitis (Figure 3). DSS-induced colitis lacks involvement of the adaptive immune system in the pathogenesis of the inflammation [24]; however, gut macrophages are thought to be main mediators because gadolinium chloride $\left(\mathrm{GdCl}_{3}\right)$, a macrophage selective inhibitor, ameliorated the mucosal damage by suppressing DSS-induced macrophagederived cytokines (IL-6, IL-1 $\beta$, and TNF $\alpha$ ) [51]. According to the results in this study, KCs do not interact with these macrophages. The detail mechanism is to be examined.

In conclusion, KCs are involved in both intrahepatic liver inflammation and extrahepatic pathogenesis of chronic arthritis, but not acute extrahepatic colitis. KCs are thought to regulate the chronic inflammation of arthritis probably through the synovial membrane phagocytes.

\section{Conclusions}

In this report, we show the possibility that liver resident macrophages (Kupffer cells), being considered to be involved in only specific local liver inflammation, are involved in extrahepatic inflammation. This finding will shed novel light on the role of this cell population in the pathogenesis of systemic diseases.

\section{Ethical Approval}

All procedures performed in studies involving animals were in accordance with the ethical standards of the institution or practice at which the studies were conducted.

\section{Conflicts of Interest}

The authors declare that they have no conflicts of interest.

\section{Acknowledgments}

Financial support for this work was provided by Musashino University (Gakushin Project).

\section{References}

[1] G. Biozzi and C. Stiffel, “The physiopathology of the reticuloendothelial cells of the liver and spleen," Progress in Liver Diseases, vol. 2, p. 166, 1965.

[2] R. S. Haltiwanger, M. A. Lehrman, A. E. Eckhardt, and R. L. Hill, "The distribution and localization of the fucose-binding lectin in rat tissues and the identification of a high affinity form of the mannose/N-acetylglucosamine-binding lectin in rat liver," Journal of Biological Chemistry, vol. 261, no. 16, pp. 7433-7439, 1986.

[3] K. Y. Helmy, K. J. Katschke Jr., N. N. Gorgani et al., "CRIg: a macrophage complement receptor required for phagocytosis of circulating pathogens," Cell, vol. 124, no. 5, pp. 915-927, 2006.

[4] K. Jung, M. Kang, C. Park et al., "Protective role of V-set and immunoglobulin domain-containing 4 expressed on kupffer cells during immune-mediated liver injury by inducing tolerance of liver T- and natural killer T-cells," Hepatology, vol. 56, no. 5, pp. 1838-1848, 2012.

[5] Y. Kimura, A. Inoue, S. Hangai et al., "The innate immune receptor Dectin-2 mediates the phagocytosis of cancer cells by Kupffer cells for the suppression of liver metastasis," Proceedings of the National Academy of Sciences of the United States of America, vol. 113, no. 49, pp. 14097-14102, 2016.

[6] D. Movita, K. Kreefft, P. Biesta et al., "Kupffer cells express a unique combination of phenotypic and functional characteristics compared with splenic and peritoneal macrophages," Journal of Leukocyte Biology, vol. 92, no. 4, pp. 723-733, 2012. 
[7] T. Okada, A. Kimura, K. Kanki et al., "Liver resident macrophages (kupffer cells) share several functional antigens in common with endothelial cells," Scandinavian Journal of Immunology, vol. 83, no. 2, pp. 139-150, 2016.

[8] N. Duarte, I. C. Coelho, R. S. Patarrão, J. I. Almeida, C. PenhaGonçalves, and M. P. Macedo, "How inflammation impinges on NAFLD: a role for Kupffer cells," BioMed Research International, vol. 2015, Article ID 984578, 11 pages, 2015.

[9] M. Hatano, S. Sasaki, S. Ohata et al., "Effects of Kupffer cell-depletion on Concanavalin A-induced hepatitis," Cellular Immunology, vol. 251, no. 1, pp. 25-30, 2008.

[10] F. Heymann, J. Peusquens, I. Ludwig-Portugall et al., "Liver inflammation abrogates immunological tolerance induced by kupffer cells," Hepatology, vol. 62, no. 1, pp. 279-291, 2015.

[11] H. Nakashima, M. Kinoshita, M. Nakashima et al., "Superoxide produced by Kupffer cells is an essential effector in concanavalin A-induced hepatitis in mice," Hepatology, vol. 48, no. 6, pp. 1979-1988, 2008.

[12] J. Schümann, D. Wolf, A. Pahl et al., "Importance of Kupffer cells for T-cell-dependent liver injury in mice," The American Journal of Pathology, vol. 157, no. 5, pp. 1671-1683, 2000.

[13] Y. Tian, W. Jochum, P. Georgiev, W. Moritz, R. Graf, and P.A. Clavien, "Kupffer cell-dependent TNF- $\alpha$ signaling mediates injury in the arterialized small-for-size liver transplantation in the mouse," Proceedings of the National Academy of Sciences of the United States of America, vol. 103, no. 12, pp. 4598-4603, 2006.

[14] H. Tsutsui and S. Nishiguchi, "Importance of kupffer cells in the development of acute liver injuries in mice," International Journal of Molecular Sciences, vol. 15, no. 5, pp. 7711-7730, 2014.

[15] N. Van Rooijen and A. Sanders, "Liposome mediated depletion of macrophages: mechanism of action, preparation of liposomes and applications," Journal of Immunological Methods, vol. 174, no. 1-2, pp. 83-93, 1994.

[16] I. Shibuya, T. Akaike, and Y. Watanabe, "Design of a temporally and spatially controlled drug delivery system for the treatment of liver diseases in mice," Hepatology, vol. 32 , no. 6, pp. 13001308, 2000.

[17] H. Sann, J. V. Erichsen, M. Hessmann, A. Pahl, and A. Hoffmeyer, "Efficacy of drugs used in the treatment of IBD and combinations thereof in acute DSS-induced colitis in mice," Life Sciences, vol. 92, no. 12, pp. 708-718, 2013.

[18] D. D. Brand, K. A. Latham, and E. F. Rosloniec, "Collageninduced arthritis," Nature Protocols, vol. 2, no. 5, pp. 1269-1275, 2007.

[19] N. Van Rooijen and A. Sanders, "Kupffer cell depletion by liposome-delivered drugs: comparative activity of intracellular clodronate, propamidine, and ethylenediaminetetraacetic acid," Hepatology, vol. 23, no. 5, pp. 1239-1243, 1996.

[20] Y. Ishijima, T. Kawamura, A. Kimura et al., “Toll-like receptor 4-dependent adjuvant activity of Kakkon-to extract exists in the high molecular weight polysaccharide fraction," International Journal of Immunopathology and Pharmacology, vol. 24, no. 1, pp. 43-54, 2011.

[21] T. Mosmann, "Rapid colorimetric assay for cellular growth and survival: application to proliferation and cytotoxicity assays," Journal of Immunological Methods, vol. 65, no. 1-2, pp. 55-63, 1983.

[22] I. Ajioka, T. Akaike, and Y. Watanabe, "Expression of vascular endothelial growth factor promotes colonization, vascularization, and growth of transplanted hepatic tissues in the mouse," Hepatology, vol. 29, no. 2, pp. 396-402, 1999.
[23] I. Koboziev, F. Karlsson, S. Zhang, and M. B. Grisham, "Pharmacological intervention studies using mouse models of the inflammatory bowel diseases: Translating preclinical data into new drug therapies," Inflammatory Bowel Diseases, vol. 17, no. 5, pp. 1229-1245, 2011.

[24] Y. Yan, V. Kolachala, G. Dalmasso et al., “Temporal and spatial analysis of clinical and molecular parameters in dextran sodium sulfate induced colitis," PLoS ONE, vol. 4, no. 6, Article ID e6073, 2009.

[25] R. W. Kinne, C. B. Schmidt-Weber, R. Hoppe et al., "Long-term amelioration of rat adjuvant arthritis following systemic elimination of macrophages by clodronate-containing liposomes," Arthritis \& Rheumatism, vol. 38, no. 12, pp. 1777-1790, 1995.

[26] P. J. Richards, A. S. Williams, R. M. Goodfellow, and B. D. Williams, "Liposomal clodronate eliminates synovial macrophages, reduces inflammation and ameliorates joint destruction in antigen-induced arthritis," Rheumatology, vol. 38, no. 9, pp. 818-825, 1999.

[27] P. Barrera, A. Blom, P. L. Van Lent et al., "Synovial macrophage depletion with clodronate-containing liposomes in rheumatoid arthritis," Arthritis \& Rheumatism, vol. 43, no. 9, pp. 1951-1959.

[28] P. L. E. M. Van Lent, A. E. M. Holthuysen, N. Van Rooijen, L. B. A. Van De Putte, and W. B. Van Den Berg, "Local removal of phagocytic synovial lining cells by clodronate- liposomes decreases cartilage destruction during collagen type II arthritis," Annals of the Rheumatic Diseases, vol. 57, no. 7, pp. 408-413, 1998.

[29] K. Greish, "Enhanced permeability and retention (EPR) effect for anticancer nanomedicine drug targeting," Methods in Molecular Biology, vol. 624, pp. 25-37, 2010.

[30] H. F. Dvorak, J. A. Nagy, J. T. Dvorak, and A. M. Dvorak, "Identification and characterization of the blood vessels of solid tumors that are leaky to circulating macromolecules," American Journal of Pathology, vol. 133, no. 1, pp. 95-109, 1988.

[31] M. Ikarashi, H. Nakashima, M. Kinoshita et al., "Distinct development and functions of resident and recruited liver Kupffer cells/macrophages," Journal of Leukocyte Biology, vol. 94, no. 6, pp. 1325-1336, 2013.

[32] I. Klein, J. C. Cornejo, N. K. Polakos et al., "Kupffer cell heterogeneity: functional properties of bone marrow-derived and sessile hepatic macrophages," Blood, vol. 110, no. 12, pp. 40774085, 2007.

[33] M. Bilzer, F. Roggel, and A. L. Gerbes, "Role of Kupffer cells in host defense and liver disease," Liver International, vol. 26, no. 10, pp. 1175-1186, 2006.

[34] A. Erhardt, M. Biburger, T. Papadopoulos, and G. Tiegs, "IL-10, regulatory $\mathrm{T}$ cells, and Kupffer cells mediate tolerance in concanavalin A-induced liver injury in mice," Hepatology, vol. 45, no. 2, pp. 475-485, 2007.

[35] S. Gehring, E. M. Dickson, M. E. San Martin et al., "Kupffer cells abrogate cholestatic liver injury in mice," Gastroenterology, vol. 130, no. 3, pp. 810-822, 2006.

[36] J. Wan, M. Benkdane, F. Teixeira-Clerc et al., "M2 Kupffer cells promote M1 Kupffer cell apoptosis: a protective mechanism against alcoholic and nonalcoholic fatty liver disease," Hepatology, vol. 59, no. 1, pp. 130-142, 2014.

[37] Q. You, L. Cheng, R. M. Kedl, and C. Ju, "Mechanism of T cell tolerance induction by murine hepatic Kupffer cells," Hepatology, vol. 48, no. 3, pp. 978-990, 2008.

[38] D. Zuo, X. Yu, C. Guo et al., "Scavenger receptor a restrains T-cell activation and protects against concanavalin A-induced hepatic injury," Hepatology, vol. 57, no. 1, pp. 228-238, 2013. 
[39] M. Tanaka, T. Nagai, Y. Tsuneyoshi et al., "Expansion of a unique macrophage subset in rheumatoid arthritis synovial lining layer," Clinical and Experimental Immunology, vol. 154, no. 1, pp. 38-47, 2008.

[40] F. Zheng, S. Put, L. Bouwens et al., "Molecular imaging with macrophage crig-targeting nanobodies for early and preclinical diagnosis in a mouse model of rheumatoid arthritis," Journal of Nuclear Medicine, vol. 55, no. 5, pp. 824-829, 2014.

[41] J. D. Milner, T. Orekov, J. M. Ward et al., "Sustained IL-4 exposure leads to a novel pathway for hemophagocytosis, inflammation, and tissue macrophage accumulation," Blood, vol. 116, no. 14, pp. 2476-2483, 2010.

[42] C. L. Elsegood, C. W. Chan, M. A. Degli-Esposti et al., "Kupffer cell-monocyte communication is essential for initiating murine liver progenitor cell-mediated liver regeneration," Hepatology, vol. 62, no. 4, pp. 1272-1284, 2015.

[43] K. Fujiu, M. Shibata, Y. Nakayama et al., "A heart-brain-kidney network controls adaptation to cardiac stress through tissue macrophage activation," Nature Medicine, vol. 23, no. 5, pp. 611622, 2017.

[44] M. Noack and P. Miossec, “Th17 and regulatory T cell balance in autoimmune and inflammatory diseases," Autoimmunity Reviews, vol. 13, no. 6, pp. 668-677, 2014.

[45] N. Komatsu, K. Okamoto, S. Sawa et al., "Pathogenic conversion of Foxp3+ T cells into TH17 cells in autoimmune arthritis," Nature Medicine, vol. 20, no. 1, pp. 62-68, 2014.

[46] E. Lubberts, M. I. Koenders, and W. B. van den Berg, "The role of $\mathrm{T}$ cell interleukin-17 in conducting destructive arthritis: lessons from animal models," Arthritis Research and Therapy, vol. 7, no. 1, pp. 29-37, 2005.

[47] S. Nakae, A. Nambu, K. Sudo, and Y. Iwakura, "Suppression of immune induction of collagen-induced arthritis in IL-17-deficient mice," Journal of Immunology, vol. 171, no. 11, pp. 61736177, 2003.

[48] P. J. De Bleser, T. Niki, V. Rogiers, and A. Geerts, “Transforming growth factor- $\beta$ gene expression in normal and fibrotic rat liver," Journal of Hepatology, vol. 26, no. 4, pp. 886-893, 1997.

[49] S. Roth, W. Gong, and A. M. Gressner, "Expression of different isoforms of TGF- $\beta$ and the latent TGF- $\beta$ binding protein (LTBP) by rat Kupffer cells," Journal of Hepatology, vol. 29, no. 6, pp. 915-922, 1998.

[50] M. Sasai, Y. Saeki, S. Ohshima et al., "Delayed onset and reduced severity of collagen-induced arthritis in interleukin-6-deficient mice," Arthritis and Rheumatism, vol. 42, no. 8, pp. 1635-1643, 1999.

[51] C. Du, P. Wang, Y. Yu, F. Chen, J. Liu, and Y. Li, "Gadolinium chloride improves the course of TNBS and DSS-induced colitis through protecting against colonic mucosal inflammation," Scientific Reports, vol. 4, article no. 6096, 2014. 


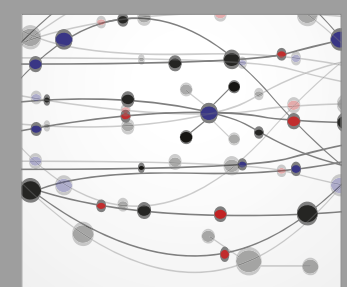

The Scientific World Journal
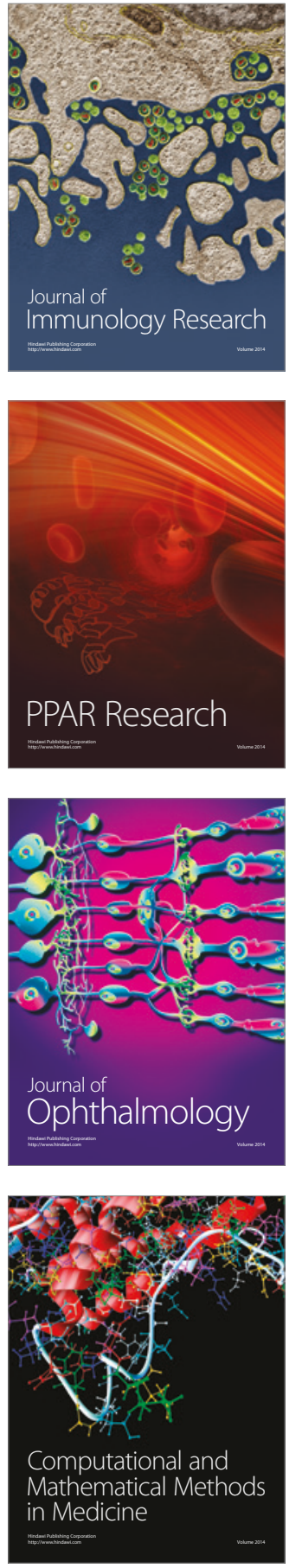

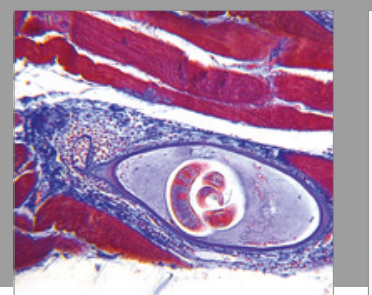

Gastroenterology Research and Practice
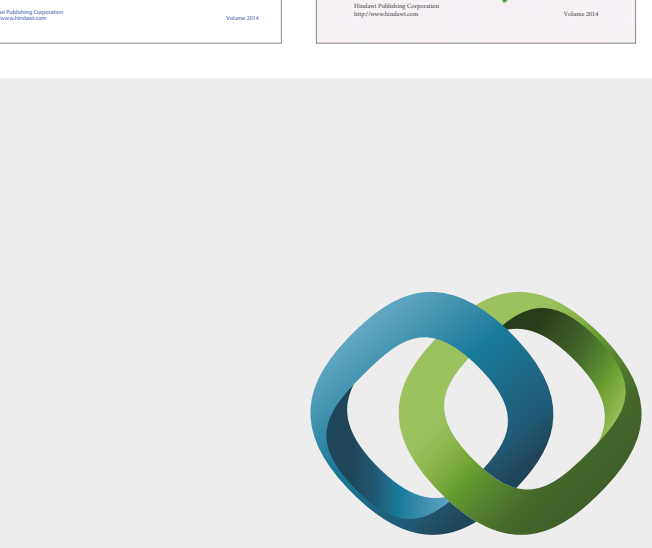

\section{Hindawi}

Submit your manuscripts at

https://www.hindawi.com
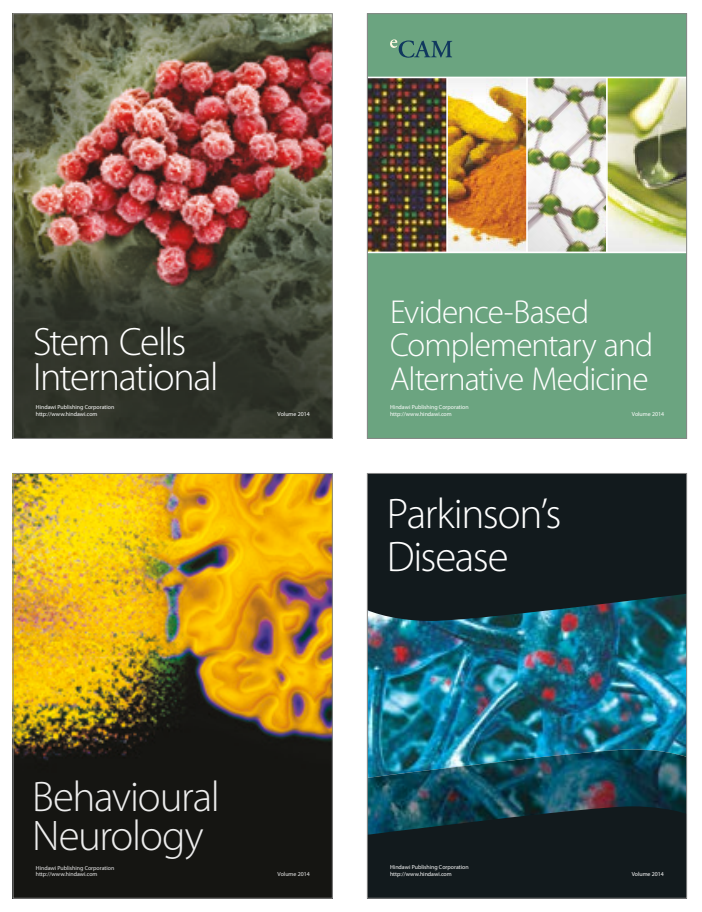
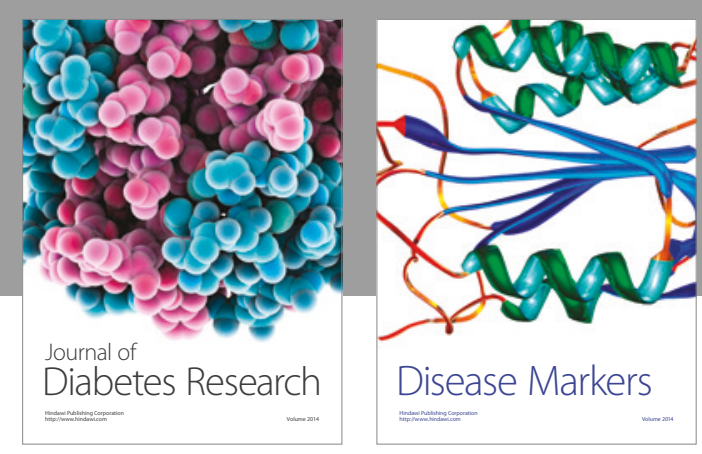

Disease Markers
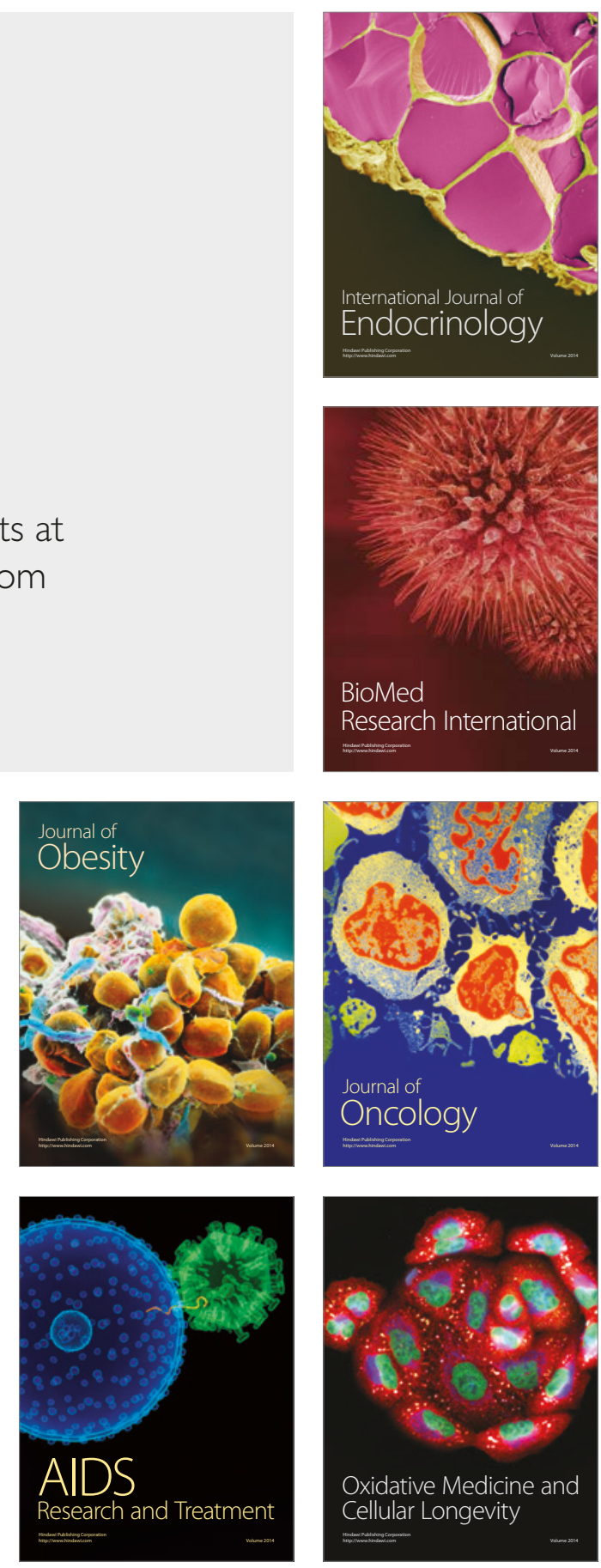